\title{
PROSPECTS FOR INCREASING THE EFFICIENCY OF TREATMENT OF PATIENTS HEAVING OF LOCALLY- DISTRIBUTED BREAST CANCER
}

\author{
Yuriy Vinnik ${ }^{1}$ \\ Yulia Belevtsova ${ }^{1}$ \\ Marina Sadchikova ${ }^{1}$ \\ ${ }^{1}$ Department of Oncological Surgery, Radiation Therapy and Palliative Care \\ Kharkiv Medical Academy of Postgraduate Education \\ 58 Amosova str., Kharkiv, Ukraine, 61176
}

\section{Abstract}

Improving the efficiency of treatment of locally distributed forms of breast cancer (BC) patients is relevant.

The aim of the study: to determine the possibility of increasing the efficiency of treatment of patients with locally distributed $\mathrm{BC}$ by supplementing it with the determination of the expression of TOP 2 alpha and beta-tubulin III genes in the primary tumor during various chemotherapy regimens.

Method. 139 patients with locally distributed BC were examined. Patients received 2-4 courses of neoadjuvant chemotherapy (NAChT) according to the TC (docetaxel + cyclophosphamide) and TAC (docetaxel + doxorubicin + cyclophosphamide) regimens, subsequent surgical intervention and 2-4 courses of AchT according to the FAC regimen. Immunohistochemical (IHCh) study of the levels of estrogen receptors (ER), progesterone (PR), epidermal growth factor (HER2) in tumors was conducted. The expression of TOP2 alpha and beta-tubulin class III genes was determined using PCR. Statistical data processing was performed using the program Stastistica for Windows 6. 0, Excel.

Results. In the third cycle of chemotherapy, patients were transferred from the TC scheme to the TAC scheme. The frequency of the FPR increased 4 times in the group of patients with a triple negative tumor subtype compared with patients with luminal B. A combination of low expression of the class III beta-tubulin gene with high expression of TOP2 alpha in tumor tissue was found in $64.5 \%$ of patients treated according to the TAC + cross over regimen and in $56.3 \%$ of cases according to the TC regimen. A change in the receptor status of MLN after NAChT was detected in some cases.

Conclusions. The combination of high expression of TOP2 alpha with low expression of class III beta-tubulins can be considered as a predictive sign of full pathomorphological response when using taxane-containing locally distributed BC. These markers can be recommended for determination in MLN (along with definition of ER, PR, HER2) in order to increase the effectiveness of AchT.

Keywords: locally distributed breast cancer, chemotherapy, estrogen, progesterone receptors, HER2, lymph nodes, topoisomerase II alpha, beta-tubulin III.

DOI: $10.21303 / 2504-5679.2020 .001286$

\section{Introduction}

Breast cancer (BC) among the oncological pathologies in women by incidence in recent years has come first in the world. More than 400 thousand women die from this disease [1]. In Ukraine, according to data of Cancer-Register of 2016-2017, [2], the incidence of breast cancer was 74.4 , mortality 30.2 per 100 thousand of the population. The average age of $80 \%$ of patients is 61 years old, and about $20 \%$ of patients are less than 50 years old (these include 30 -year-old and 40 -year-old women). The high incidence of breast cancer and mortality from this pathology, the high recurrence rate after standard treatment requires further study of ways to increase the efficiency of treatment for breast cancer and makes this issue relevant.

Current recommendations for the treatment of $\mathrm{BC}$ are based on an understanding of its biological heterogeneity, which determines the need to isolate molecular biological types for adequate individualized therapy. Based on the immunohistochemical (IHCh) analysis of estrogen receptors (ER) and progesterone (PR), epidermal growth factor receptor 2 (HER2), some cytokeratins, the expression level of the Ki-67 proliferation marker, a breast cancer classification was created, 
according to which $\mathrm{BC}$ variants differ in prognosis and sensitivity to various types of drug therapy $[1,3]$. The frequency of detection of various molecular types of $\mathrm{BC}$, determined on the basis of IHCh evaluation of the expression of ER, PR and HER2/neu, is not the same. Moreover, luminal types are associated with a less aggressive course and a good prognosis in comparison with HER2 + and basal-like types [4]. The presence of estrogen and progesterone receptors in a breast tumor correlates with a good prognosis of relapse-free and overall patient survival [5]. The results of IHCh studies of BC have opened up prospects for the use of modern methods of complex and combined treatment, the use of targeted drugs.

Based on immunohistochemical (IHC) analysis of estrogen and progesterone receptors, epidermal growth factor receptor HER2, some cytokeratins, the expression level of the Ki-67 proliferation marker, a breast cancer classification was created, according to which antibodies of breast cancer differ in prognosis and sensitivity to various types of drug therapy $[1,3]$.

The frequency of detection of various molecular types of BC, determined on the basis of IHCh evaluation of the expression of ER, PR and HER2/neu, is not the same. Moreover, luminal types are associated with a less aggressive course and a good prognosis in comparison with HER2 + and basal-like types [4]. The presence of estrogen and progesterone receptors in a breast tumor correlates with a good prognosis of relapse-free and overall patient survival [5]. The results of IHCh studies of $\mathrm{BC}$ have opened up prospects for the use of modern methods of complex and combined treatment, the use of targeted drugs. At the same time, the issues of hormonal activity of metastatic lymph nodes, their production of various signal molecules (which can be especially informative with neoadjuvant therapy) have not been studied enough. It should be noted that certain recommendations regarding the need for immunomorphological studies of LN in the presence of metastases in them do not exist. Moreover, metastatic lesion of regional LN in BC is the most significant prognosis [6, 7]. Therefore, the issue of accurate and detailed diagnosis of regional metastases is of fundamental importance. To date, morphological and IHC studies can accurately diagnose metastases in regional LN in patients with breast cancer, however, such a combined approach is time-consuming, requires considerable time, and the result is not always objective.

Theoretically, the cellular elements of metastases are the most aggressive clone of tumor cells, and it is advisable to pay attention to them as potentially circulating in the body and being the target for adjuvant therapy, since the primary tumor has already been removed.

The use of neoadjuvant chemotherapy (NAChT) of locally distributed BC and its inflammatory forms, the purpose of which is to transfer the tumor from inoperable to operable, reduce the stage of the disease, evaluate the pathological response, allows you to recognize the sensitivity to a particular drug at the preoperative stage $[8,9]$. According to a large array of studies $[10,11]$, the primary tumor and micrometastases have similar sensitivity to ChT drugs.

Therefore, with the ineffectiveness of neoadjuvant systemic therapy, a similar response to treatment with the same drugs in the adjuvant mode can be predicted. However, according to [12] and based on our own studies [13], differences in the immunophenotype of cells in primary tumors and their metastases were revealed. The results of our previous IHCh and morphological studies $[13,14]$ showed that in the process of conducting NAChT not only the loss of the corresponding receptors occurs, but also the transformation of cells with the appearance of new phenotypes. The appearance of new receptors (ER, HER-2) occurs much less frequently than the loss of the same receptors under the influence of neoadjuvant therapy (NAChT). The differences between the primary tumor and metastases in the LN can be explained from the standpoint of the theory of cancer stem cells (CSC) and epithelial-mesenchymal transformation (EMT). The use of a comprehensive and combined treatment approaches, taking into account not only the morphobiological type of the primary tumor, but also regional lymph nodes, opens the way to individualizing treatment, reducing the number of relapses, and increasing the five-year relapse-free survival rates.

It is known, that modern NAChT is characterized by a number of drawbacks, including the empirical choice of its purpose, a decrease in the quality of life of patients, and a low therapeutic effect. Therefore, the rejection of deliberately ineffective treatment may be the best solution to the problem [15]. In this regard, it becomes appropriate to search for markers that can predict the 
response to chemotherapy in advance, reduce the frequency of side effects, and reduce the cost of treatment due to the more reasonable use of drugs $[16,17]$.

Knowledge of the molecular genetic characteristics of not only the primary tumor, but also regional LN can individualize the choice of cytostatic and reduce the risk of side effects [18]. Among these characteristics described in the scientific literature are: topoisomerase II alpha (TOR2 alpha) (a complex protein necessary for DNA replication, the main target of doxorubicin, which inhibits TOR2 alpha), and beta-tubulin III protein (responsible for sensitivity to taxane formulations that stabilize its HDF-bound form). This leads to the blocking of depolymerization, stimulation of tubulin polymerization due to the absence of GTP, stops the cell cycle and activates apoptosis. The data on the predictive and prognostic value of the expression of the TOP2 alpha and beta tubulin III genes for patients with locally distributed BC are contradictory [18, 19]. According to [20, 21], overexpression of this protein in tumors in the treatment of ovarian cancer (RV) and lung cancer (RL) with paclitaxel is an unfavourable prognostic factor [22, 23].

In connection with the foregoing, the aim of this study was to determine the possibility of increasing the effectiveness of treatment of patients with locally distributed BC by supplementing it with the determination of the expression of topoisomerase II alpha (TOP2 alpha) and beta-tubulin III genes in the primary tumor during various chemotherapy regimens. To evaluate the possibility of determining these parameters in metastatic lymph nodes after cytoreductive surgery to individualize adjuvant chemotherapy.

\section{Materials and Methods}

139 patients with a diagnosis of breast cancer aged 29 to 64 years old, who were from 2014 to 2018, were examined. They were undergoing treatment at the chemotherapy department of the Kharkiv Regional Clinical Oncology Center and the city clinical multidisciplinary hospital No. 17.

The criteria for inclusion of women in the survey group were: age over 18 years old, histologically confirmed locally distributed BC (T1-2N2-3M0; T4N0-3M0); the presence of measurable foci of the tumor; known status of estrogen receptors (ER) and/or progesterone (PR), as well as epidermal growth factor (HER2/neu); consent of the patient with breast cancer to receive a complex of examination and treatment. The exclusion criteria from the examination were: conducting therapy in other medical institutions; severe neurological pathology; the presence of another active oncopathology; pregnancy; uncontrolled hypertension, a high degree of risk of uncontrolled arrhythmia; the following changes in laboratory parameters (creatinine $>2.0 \mathrm{mg} / \mathrm{dl}$; ALT, AST or serum bilirubin 2.5 times higher than normal (total serum bilirubin $>27.34 \mu \mathrm{mol} / \mathrm{l}$ ); hemoglobin $<9 \mathrm{~g} / \mathrm{l}$; platelets less than 100000/ $\mu$; leukocytes less than 2.109/1 mental illness, drug or alcohol addiction; lack of patient consent.

The studies were carried out in accordance with the Declaration of Helsinki; the procedures were approved by the local ethics committee of Kharkiv medical academy of postgraduate education, protocol No. 5, 12.11.2019. Informed consent for the participation was obtained from all the patients.

To determine the biological subtype of the tumor (luminal A; luminal B; three times negative HER2 +), ER and PR levels, HER2/neu expression, and the degree of histological malignancy $(\mathrm{G})$ were determined using the IHC method. Additionally, the amplification of the HER2 gene was determined by the FISH method.

The complex treatment measures for the examined patients included: conducting 2-4 courses of NAChT according to the TC (docetaxel + cyclophosphamide) and TAC (docetaxel + doxorubicin + cyclophosphamide) regimens, subsequent surgery and 2-4 courses of adjuvant therapy according to the FAC scheme. Radiation or hormone therapy was prescribed according to indications. Every 2 cycles the clinical response using physical and instrumental methods were studied. When stabilization occurs after two cycles of NACT according to the TC regimen, patients were transferred to the TAC group. In this case, stabilization was considered as an insufficient response to the NACT.

The clinical evaluation of NAChT was carried out according to the following criteria: complete disappearance of the primary tumor in the mammary gland and regional LN; reduction in the 
size of the tumor by $50 \%$ or more; stabilization - criteria insufficient to achieve partial regression; disease progression - an increase in tumor size by more than $25 \%$.

The degree of drug pathomorphosis was evaluated according to the classification by I. D. Miller, S. Payne (2002).

Monoclonal rabbit antibodies (MKAB) were used for IHC against estrogen receptors SP1 Spring Bio 1:250, against progesterone receptors SP2 Spring Bio 1:350, against HER2/neu V9 Roche/Ventana rtu. 1:100. To determine the expression of topoisomerase II-alpha and betatubulin class III genes, real-time PCR was used using Taq Man-samples using a BioRad CFX96 Real time PCR detection System (USA). Expression indices of less than 20 percentiles were categorized as "low", and more than 80 percentiles were "high".

Statistical processing of the obtained data was carried out using the program Stastistica for Windows 6. 0, statistical package R and the spreadsheet processor Excel. Differences were considered significant at $\mathrm{p}<0.05$.

\section{Results}

Comparison of treatment results for locally distributed BC patients according to TAC and TC schemes showed the advantage of the first of the listed options (i. e. TAC). At the same time, there were no significant differences between taxane-containing chemotherapy with the addition of anthracyclines and without them with a complete pathomorphological response (T0N0) due to the fact that in the third cycle of PCT, patients were transferred from the TC scheme to the TAC scheme, since the stabilization found in them was regarded as insufficient answer.

Studies have shown that the frequency of the full pathomorphological response increased 4 times in the group of patients with a triple negative tumor subtype compared with BC patients with luminal B. Moreover, with luminal A it was $8.5 \%$ (confidence interval (CI 2.7-17.0), with luminal B - $6.7 \%$ (CI 0.5-18.8), with HER2 + 23.8 \% (CI 7.7-45.3), with a triple negative subtype $-25.8 \%$ (CI 11.7-43.1).

A significant pathomorphological response was observed in 11 patients with luminal A subtype (4+5 tbsp. According to Miller-Raul), which amounted to 8.6\% (CI 9.7-29.7); in 5 patients with a luminal B subtype (16.7 \% CI 5.4-32.6); in 9 patients with HER2 + type $42.9 \%$ (CI 21.865.4); in 12 patients in the group of thrice negative subtype of breast cancer, 38.7 \% (CI 22.0-57.0).

In patients with HER $2+$ and thrice-negative breast cancer, when anthracycline or taxane was included in ChT, a higher incidence of CDPT (complete disappearance of the primary tumor) and a significant pathomorphological tumor response (4+5 st. according to Miller-Raul) were observed compared with the luminal subtypes A and B. It should be noted that complete disappearance of the primary tumor and a significant pathomorphological response were considered by us in each group as criteria for the effectiveness of taxane-containing chemotherapy.

The determination of beta-tubulin III expression in the tumor showed low results in 34 patients $(58.6 \%)$ and high results in $24(41.4 \%)$ patients. In the group of patients with low expression of class III beta-tubulin in the tumor, a significant pathomorphological response was achieved in $18(23.1 \%)$ women, a significant degree of pathomorphological response was achieved in 16 patients $(39.7 \%)$. At the same time, in the group of patients with low expression of class III beta-tubulin, CDPT was observed in 18 cases $(23.1 \%)$, and in the group with their high expression, only in one patient $(\mathrm{p}<0.001)$.

In the group of patients with low expression of class III beta-tubulin who received chemotherapy according to the TAC + cross over (TC-TAC) regimen, CDPT was observed in 10 patients $(20.8 \%)$, and in the group with high expression, in one patient $(2.4 \%)(0.0-9.6)$ at $\mathrm{p}<0.005$.

In patients with low expression of beta-tubulin III who received chemotherapy according to the TS regimen, a significant pathomorphological response was detected in 8 cases (26.7 \%) (CI 12.1$44.4)$, and in the group with high expression, only in 1 patient $(\mathrm{p}<0.005)$. The obtained results allow us to state that low expression of the class III beta-tubulin gene in tumor tissue in breast cancer is a reliable predictive sign of the achievement of MTCT in response to taxane-containing chemotherapy.

The predictive value of topoisomerase II-alpha as a marker of tumor sensitivity to anthracycline antibiotics was also studied in a group of patients who received chemotherapy with 
TAC + cross over. In patients with breast cancer with low gene expression of topoisomerase II-alpha in response to NACT with the inclusion of anthracycline antibiotics, a significant pathomorphological response was observed in 1 patient (1.6\%), and in the group with high expression, in $18(24.7 \%)(\mathrm{p}<0.001)$. In the group of patients with high expression of topoisomerase, the II-alpha pathomorphological response was achieved in 33 (45.2\%) women (33.8-56.8) with $\mathrm{p}<0.001$.

When establishing high expression of topoisomerase II-alpha in combination with low expression of class III beta-tubulin, a significant pathomorphological response in the group of breast cancer patients treated according to the TAC + cross over scheme was achieved in 20 cases $(64.5 \%)$, in the group of patients treated according to the TC-scheme in 9 women (56.3\%) $(30.0-80.7)$ at $\mathrm{p}=0.58$.

The combination of high expression of topoisomerase II-alpha and low expression of class III beta-tubulin in relation to CDPT showed that in the group of patients who received NAChT according to the TAC + cross over scheme, the 5th degree of pathomorphological response was achieved in 10 cases $(32.3 \%)(16.7-50.2)$, and according to the TC scheme, in 7 cases $(43.8 \%)$ (19.3-70.0).

Thus, the combination of high expression of topoisomerase II-alpha with low expression of class III beta-tubulin can be considered as a predictive sign of achieving CDPT when using taxane-containing chemotherapy. Therefore, the molecular markers mentioned above should be included in the diagnostic complex for evaluating the efficacy of NAChT in patients with locally distributed BC and can be recommended for determination in LN (along with ER, PR, HER2) after cytoreductive surgery to plan effective AChT.

\section{Discussion}

Discussing the results with the data of scientific literature, we can conclude that one of the main problems of tumor resistance in locally distributed BC to therapy is the change in its biology during progression or under the influence of the therapy $[13,14]$. During the implementation of the metastatic cascade, cancer cells, losing a set of epithelial antigens and acquiring a set of mesenchymal (epithelial-mesenchymal transformation-EMT), can change their initial phenotype (for example, lose steroid hormonal receptors or epidermal growth factor receptors) [24]. This explains the fact of different chemosensitivity of the primary tumor and its lymphogenous metastases, which must be taken into account when conducting adjuvant treatment. Individualization of antitumor therapy involves treatment not only taking into account the molecular status of the primary tumor, but also taking into account the phenotype of the cells of its metastases [19].

It should be noted that in some cases, the use of approaches to increase the efficiency of the treatment of hormone-dependent tumors of other locations, for example, locally distributed ovarian cancer, may become promising for solving the same problem with respect to breast cancer. Thus, according to [25], frequent relapses of the disease after treatment and a high mortality rate in patients with stage III-IV ovaries worldwide can be explained from the standpoint of the theory of tumor stem cells based on the study of prognostic tumor markers, which allows us to adjust the treatment strategy. The results of the determination of Ki-67 made it possible to supplement the clinical signs of the efficiency of NAChT in OC III-IV stages, indicating a decrease in this indicator in the tumor after NAChT by almost 4 times, which was accompanied by a decrease in CA125. In patients with a locally advanced tumor process, an opportunity arose for cytoreductive surgery.

At the same time, an increase in the expression of bcl-2, a decrease in the expression of cytokeratin-7 in $37 \%$ of patients and E-cadherin in tumors after NAChT is a sign of a poor prognosis of the further course of OC, which can be explained from the perspective of the theory of CSC, responsible for maintaining oncogenesis and heterogeneity tumors. The rupture of intercellular bonds in accordance with a decrease in the expression of E-cadherin can be one of the factors of epithelial-mesenchymal transformation (EMT), cell de-differentiation, acquisition of an CSC phenotype by a tumor cell, and transition of cells to a mesenchymal phenotype. Such cells have an aggressive malignant potential that affects survival. At the same time, a high degree of expression of cytokeratin-7 in $63 \%$ of patients with OC of III-IV art. gives hope for the differentiation of 
tumor cells. Despite the effectiveness of the influence of NPHT on the treatment of late stage OC, the threat of relapse of the OC due to the formation of CSC and the threat of EMT remains in the patient's body. A promising approach to the development of new methods for the treatment of late stage ovaries can be the prevention of EMT and, in particular, the loss of E-cadherin.

The question of creating a complex of quantitative diagnostic criteria for the efficiency of NAChT and AHT, criteria for evaluating EMT, and prognosis of the course of locally distributed $\mathrm{BC}$ is being discussed.

Study limitations. Thus, the solution to the problem of increasing the efficiency of treatment for BC is not limited to determining the expression of TOP 2 alpha and beta-tubulin III genes in the primary tumor during various chemotherapy regimens and metastatic LN to individualize adjuvant chemotherapy and has prospects.

Prospects for further research. Prospects for further research: promising continuation of research may be the determination of tumor markers of epithelial-mesenchymal transformation in a tumor. Such studies will help to identify patients with breast cancer with a poor prognosis and to develop ways to prevent epithelial-mesenchymal transformation.

\section{Conclusion}

The receptor status of metastatic lymph nodes in patients having locally distributed BC may change under the influence of chemotherapy and may differ from the receptor status of the primary tumor.

The combination of high expression of topoisomerase II-alpha with low expression of class III beta-tubulin can be considered as a predictive sign of the achievement of full pathomorphological response when using taxane-containing chemotherapy in patients having locally distributed BC.

One of the ways to increase the treatment efficiency of locally distributed BC can be considered not only the determination of the expression of topoisomerase II alpha and beta-tubulin class III in the primary tumor and metastatic LN, along with the determination of ER, PR, HER2, which allows to increase the efficiency of NAChT and AHT.

The determination of molecular markers characterizing epithelial-mesenchymal transformation in a tumor is a promising approach to increasing the effectiveness of the treatment of locally distributed $\mathrm{BC}$ due to inhibition or prevention of this process.

\section{Conflict of interest}

The authors declare there is no conflict of interest.

\section{References}

[1] Sadchikova, M. V., Siniavina, L. V., Otchenash, N. M. (2019). Role of hormone therapy in treatment of early breast cancer. International Medical Journal, 25 (2), 40-44.

[2] National Cancer Register Bulletin- Cancer in Ukraine, 2016-2017 (2018). Kyiv, 19, 44-45. Available at: http://www.ncru.inf. ua/publications/BULL_19/PDF_E/44-45\%20shm.pdf

[3] Semiglazov, V. F. (2013). New approaches to the treatment of breast cancer. Oncology Issues, 59 (3), 288-291.

[4] Semiglazov, V. F., Bozhok, A. A., Semiglazova, T. Yu., Vasiliev, A. G., Manihas, A. G., Semiglazov, V. V. et. al. (2013) HER2-positive breast cancer: standard and dual targeted treatment. Oncology Issues, 59 (3), 341-346.

[5] Semiglazova, T. Yu., Klimenko, V. V., Filatova, L. V., Chubenko, V. A., Krivorotko, P. V. et. al. (2013). Markers of the effectiveness of preoperative taxane-containing chemotherapy for locally advanced breast cancer. Oncology Issues, 59 (3), 363-368.

[6] Cianfrocca, M., Goldstein, L. J. (2004). Prognostic and Predictive Factors in Early-Stage Breast Cancer. The Oncologist, 9 (6), 606-616. doi: http://doi.org/10.1634/theoncologist.9-6-606

[7] Soerjomataram, I., Louwman, M. W. J., Ribot, J. G., Roukema, J. A., Coebergh, J. W. W. (2007). An overview of prognostic factors for long-term survivors of breast cancer. Breast Cancer Research and Treatment, 107 (3), 309-330. doi: http://doi.org/ 10.1007/s10549-007-9556-1

[8] Bershtein, L. M. (2013). Endocrinology of breast cancer as a heterogeneous disease: a decade after the Millennium. Oncology Issues, 59 (3), 292-298.

[9] Semiglazov, V. F., Krivorotko, P. V., Dashyan, G. A., Zhiltsova, E. K., Ivanova, O. A., Ivanov, V. G. et. al. (2017). Neoadjuvant systemic therapy of breast cancer (retrospective and prospective analysis). Oncology Issues, 63 (2), 256-260. 
[10] Dowlatshahi, K., Witt, T. R. et. al. (2000). Detection of occult micrometastases by $0.25 \mathrm{~mm}$ sectioning and cytokeratin staining of sentinel nodes in early breast cancer. ASCO, 305.

[11] Klimenko, V. V. (2015). Molecular markers of the effectiveness of preoperative chemotherapy for locally advanced breast cancer. FGBU "SII of Oncology by N. N. Petrov", 22.

[12] Semiglazov, V. F., Semiglazov, V. V., Dashyan, G. A. (2010). Problems of surgical treatment of breast cancer (The usefulness of a biopsy of signal lymph nodes. Local surgical treatment. Journal of Practical Oncology, 11 (4), 217-220.

[13] Vinnik, Yu. A., Belevtsova, Yu. Yu. (2018) Biological features of local -advanced breast cancer in primary tumor and metastases. Kharkiv surgical school, 5-6 (92-93), 56-59.

[14] Belevtsova, Yu. Yu. (2019). Individualization of neoadjuvant rherapy in patients with locally-distributed breast cancer. International medical journal, 1, 65-69.

[15] Wein, L., Luen, S. J., Savas, P., Salgado, R., Loi, S. (2018). Checkpoint blockade in the treatment of breast cancer: current status and future directions. British Journal of Cancer, 119 (1), 4-11. doi: http://doi.org/10.1038/s41416-018-0126-6

[16] Robert, J., Morvan, V. L., Smith, D., Pourquier, P., Bonnet, J. (2005). Predicting drug response and toxicity based on gene polymorphisms. Critical Reviews in Oncology/Hematology, 54 (3), 171-196. doi: http://doi.org/10.1016/j.critrevonc.2005.01.005

[17] Chabner, B. A., Roberts, T. G. (2005). Chemotherapy and the war on cancer. Nature Reviews Cancer, 5 (1), 65-72. doi: http:// doi.org/10.1038/nrc1529

[18] Guiu, S., Michiels, S., André, F., Cortes, J., Denkert, C., Di Leo, A. et. al. (2012). Molecular subclasses of breast cancer: how do we define them? The IMPAKT 2012 Working Group Statement. Annals of Oncology, 23 (12), 2997-3006. doi: http:// doi.org/10.1093/annonc/mds586

[19] Coon, J. S., Marcus, E., Gupta-Burt, S. et. al. (2002). Amplification and overexpression of topoisomerase II alpha predict response to anthracycline-based therapy in locally advanced breast cancer. Clinical Cancer Research, 8 (4), 1061 - 1067.

[20] Mozzetti, S., Ferlini, C., Concolino, P. et. al. (2005). Class III beta-tubulin overexpression is a prominent mechanism of paclitaxel resistance in ovarian cancer patients. Clinical Cancer Research, 11 (1), 298-305.

[21] Vincenzi, B., Cesa, A. L., Santini, D., Schiavon, G., Grilli, C., Graziano, F., Tonini, G. (2004). Predictive factors for response to chemotherapy in colorectal cancer patients. Critical Reviews in Oncology/Hematology, 52 (1), 45-60. doi: http:// doi.org/10.1016/j.critrevonc.2004.05.005

[22] Knoop, A. S., Knudsen, H., Balslev, E., Rasmussen, B. B., Overgaard, J., Nielsen, K. V. et. al. (2005). Retrospective analysis of topoisomerase IIa amplifications and deletions as predictive markers in primary breast cancer patients randomly assigned to cyclophosphamide, methotrexate, and flu-orouracil or cyclophosphamide, epiru-bicin, and fluorouracil: Danish Breast Cancer Cooperative Group. Journal of Clinical Oncology, 23 (30), 7483-7490.

[23] Urano, N., Fujiwara, Y., Doki, Y., Kim, S., Miyoshi, Y., Noguchi, S., ... Monden, M. (2006). Clinical significance of class III $\beta$-tubulin expression and its predictive value for resistance to docetaxel-based chemotherapy in gastric cancer. International Journal of Oncology, 28 (2), 375-381. doi: http://doi.org/10.3892/ijo.28.2.375

[24] Liu, L., Gerson, S. L. (2006). Targeted modulation of MGMT: clinical implications. Clinical Cancer Research, 12 (2), 328-331. doi: http://doi.org/10.1158/1078-0432.ccr-05-2543

[25] Prokopyuk, A. V., Knyazyeva, M. V. (2019). About epithelial-mesenchymal relationships in tumors of patients with stage III-IV ovarian cancer. White Nights. Saint Petersburg: Ed. ANNMO. Oncology Issues, 70. 